IAC-06-A2.3.07

\title{
SUBCOOLED POOL BOILING ON THIN WIRE IN MICROGRAVITY
}

\author{
J. F. Zhao*, S. X. Wan, G. Liu, N. Yan, W. R. Hu \\ National Microgravity Laboratory/CAS, Institute of Mechanics, Chinese Academy of Sciences. 15 \\ Beisihuan Xilu, Beijing 100080, China \\ ('E-mail address of the corresponding author: jfzhao@imech.ac.cn)
}

\begin{abstract}
:
The present paper reports a new set of experimental data of subcooling pool boiling on a thin wire in microgravity aboard the $22^{\text {nd }}$ Chinese recoverable satellite using the temperature-controlled pool boiling (TCPB) device, which was developed to perform pool boiling heat transfer studies in both normal and microgravity. The results of the experiments in normal gravity before and after the flight experiment were also presented in the present paper, which were compared with those in microgravity. In all cases, the bulk fluid was R113 at $0.1 \mathrm{MPa}$ and subcooled by $26^{\circ} \mathrm{C}$ nominally. A thin platinum wire of $60 \mu \mathrm{m}$ in diameter and $30 \mathrm{~mm}$ in length is simultaneously used as heater and thermometer. Three modes of heat transfer, namely single-phase natural convection, nucleate boiling, and two-mode transition boiling, are observed in the experiment aboard the $22^{\text {nd }}$ Chinese recoverable satellite and those in normal gravity before and after the flight experiment, while only the characteristics of nucleate pool boiling is analyzed in the present paper. It's found that the heat transfer of nucleate pool boiling is slightly enhanced in microgravity comparing with those in normal gravity. It's also found that the correlation of Lienhard and Dhir (1973) can predict the CHF with good agreement, although the range of the dimensionless radius is extended by three or more decades above the originally set limit. Three critical bubble diameters are observed in microgravity, which divide the observed vapor bubbles into four regimes with different sizes. Considering the Marangoni effect, a qualitative model is proposed based on the model of Lee (1992) to reveal the mechanism underlying the bubble departure processes and a quantitative agreement can also be acquired.
\end{abstract}

Keywords: nucleate pool boiling, microgravity, subcooling, bubble departure diameter

\section{Introduction}

Pool boiling in microgravity has become an increasing significant subject for investigation, since many potential applications exist in space and on planetary neighbors due to its high efficiency. However, the investigation in microgravity suffers for unique and stringent constraints in terms of size, power and weight of experimental apparatuses, and of number and duration of the experiments. Thus, only a partial and in some aspects contradictory knowledge of microgravity boiling has been attained so far. On 
the progress in this field, several comprehensive reviews are available. They are authored by Straub (2001), and Di Marco \& Grassi (2002), among many others.

Boiling is a very complex and illusive process because of the interrelation of numerous factors and effects as the nucleate process, the growth of the bubbles, the interaction between the heater's surface with liquid and vapor, the evaporation process at the liquid-vapor interface, and the transport process of vapor and hot liquid away from the heater's surface. For a variety of reasons, fewer studies have focused on the physics of the boiling process than have been tailored to fit the needs of engineering endeavors. As a result, the literature has been flooded with the correlations involving several adjustable, empirical parameters. These correlations can provide quick input to design, performance, and safety issues and hence are attractive on a short-term basis. However, the usefulness of the correlations diminishes very quickly as parameters of interest start to fall outside the range of physical parameters for which the correlations were developed. Thus, the physics of the boiling process itself is not properly understood yet, and is poorly represented in the most correlations, despite of almost seven decades of boiling research.

The present work is a research effort on subcooled pool boiling heat transfer both in normal gravity on Earth and in microgravity aboard the $22^{\text {nd }}$ Chinese recoverable satellite. A temperature-controlled pool boiling (TCPB) device has been developed to perform pool boiling heat transfer studies in both normal and microgravity. The results of the experiments in normal gravity before and after the flight experiment were also presented in the present paper, which were compared with those in microgravity.

\section{Experimental Facility}

A temperature-controlled pool boiling (TCPB) device (Fig. 1) has been developed to perform such studies both in normal gravity on Earth and in microgravity aboard the $22^{\text {nd }}$ Chinese recoverable satellite. Detailed description of the experimental facility can be read in Wan et al. (2003).

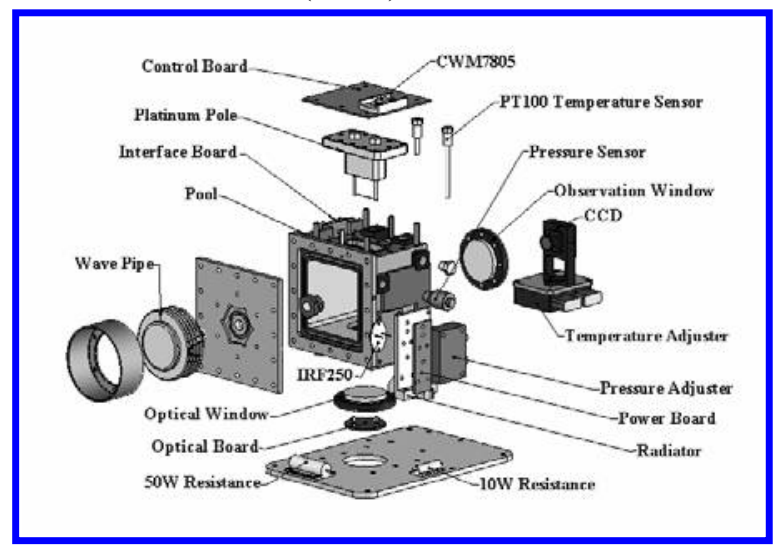

Fig. 1 Solid decomposition diagram of pool and accessories

A platinum wire of $60 \mu \mathrm{m}$ in diameter and $30 \mathrm{~mm}$ in length is simultaneously used as the heater and thermometer, with the advantage that because of its low thermal capacity, it reacted almost without any delay on changes in temperature and heat transfer, respectively. The ends of the wire are soldered with copper poles of $3 \mathrm{~mm}$ in diameter to provide a firm support for the wire heater and low resistance paths for the electric current. The heater resistance, and thus the heater temperature, are kept constant by a feedback circuit similar to that used in constant-temperature hot-wire anemometry.

The working fluid is degassed R113 with nominally a volume of $700 \mathrm{ml}$. Two platinum resistance thermometers with a range of $0 \sim 60$ ${ }^{\circ} \mathrm{C}$ are used to measure the bulk temperature of the fluid in the boiling chamber, which are calibrated to within $0.25^{\circ} \mathrm{C}$. The absolute pressure within the boiling chamber is measured using a pressure transducer with a range of $0 \sim$ 
$0.2 \mathrm{MPa}$ and an accuracy of $0.25 \% \mathrm{FS}$ (full scale). Two LEDs (light-emitting diode) are used to light the boiling chamber through a window at the chamber bottom. A CCD video camera is used to obtain images of the motion of vapor bubble or film around the heater, which is recorded by a VCR at a speed of 25 FPS (frame per second).

The voltages across the heater and a reference resistance, which is used to measure the electric current through the heater are sampled at $20 \mathrm{~Hz}$, and the outputs of the pressure transducer and the platinum resistance thermometers are sampled at $1 / 3 \mathrm{~Hz}$. A sample rate of $1000 \mathrm{~Hz}$ for every data channel is used in ground experiments. The analysis shows that the uncertainty of the heater temperature can be significantly reduced by using high sample rate (less than $3^{\circ} \mathrm{C}$ ), while the influence of the sample rate on the uncertainty of heat flux is negligible. The uncertainty of heat flux is less than $24 \mathrm{~kW} / \mathrm{m}^{2}$ in space experiment and $21 \mathrm{~kW} / \mathrm{m}^{2}$ in ground experiments, respectively.

\section{Experiment Results}

Detailed experiment conditions of the space and ground experiments are listed in Table 1. The normal pressure and subcooling of the bulk liquid are $0.1 \mathrm{MPa}$ and $26^{\circ} \mathrm{C}$.

\begin{tabular}{llll}
\hline & $\begin{array}{l}\text { Pressure } \\
(\mathrm{kPa})\end{array}$ & $\begin{array}{l}T_{l} \\
\left({ }^{\circ} \mathrm{C}\right)\end{array}$ & $\begin{array}{l}\Delta T_{\text {sub }} \\
\left({ }^{\circ} \mathrm{C}\right)\end{array}$ \\
\hline $\begin{array}{l}\text { Ground experiment } \\
\text { before the flight }\end{array}$ & 101.2 & 22.1 & 25.5 \\
\hline Space experiment & 101.0 & 21.3 & 26.2 \\
\hline $\begin{array}{l}\text { Ground experiment } \\
\text { after the flight }\end{array}$ & 101.4 & 20.5 & 27.1 \\
\hline
\end{tabular}

Table 1 Experiment conditions of space experiment and ground experiments

The first heat transfer mode is single-phase natural convection both in normal gravity and in microgravity. The comparison between experiment data and the common used the relation of Kuehn \& Goldstein (1976) is shown in Fig. 2. An agreement is quite evident between the results of ground experiments and the prediction, which proves the credibility of the experimental facility. Comparing the results of space experiments with the predictions, it's indicated that the residual gravity during the space experiment is between $10^{-3}$ to $10^{-5} \mathrm{~g}_{0}$, which is equivalent to that given by the satellite establishment.

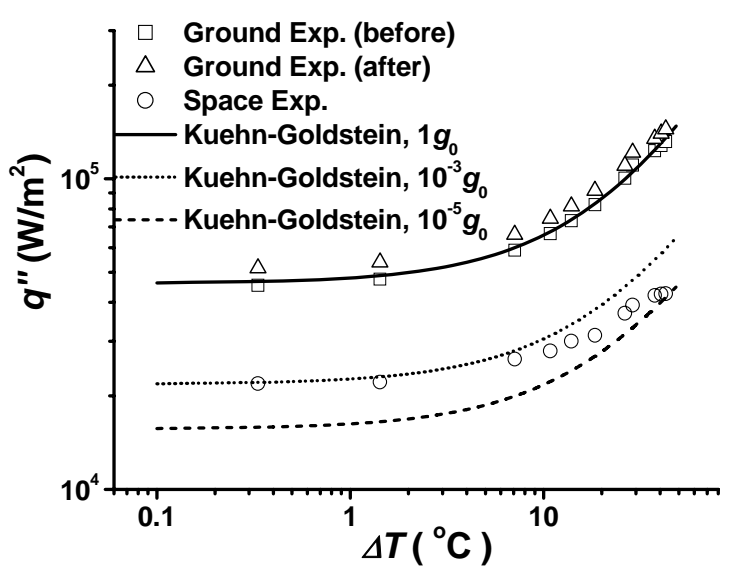

Fig.2 Single phase convection

The onset of boiling occurs at the $16^{\text {th }}$ set-point as two-mode transition boiling both in the space experiment and in ground experiments. The onset-boiling temperature is $83.2^{\circ} \mathrm{C}$, which is independent, or at least, dependent much weakly on gravity. Detailed analysis on the data of two-mode transition boiling will be presented elsewhere in the future.

Fig. 3 shows the variety of heat flux near the CHF in different gravity levels. Steady nucleate boiling is observed with high heat flux in the $42^{\text {nd }}$ set-point of the heater temperature $\left(T_{\mathrm{W}, 42}\right)$. When the set-point of the heater temperature changed upward from $T_{\mathrm{W}, 42}$ to $T_{\mathrm{W}, 43}$ in the space experiments, the heat flux is ascending to another steady nucleate boiling state with a higher heat flux. This state, however, 
can not be kept after about 1 second. A quick drop is observed which is caused by the appearance of two-mode transition boiling. From the video record, it's found that a middle bubble on the right side of the heater was coalesced by a large departed bubble, the vibration due to the coalescence of bubbles leads to the fact that a vapor film covers the heater on the location of the middle bubble. Therefore, the CHF should locate between $T_{\mathrm{W}, 42}$ and $T_{\mathrm{W}, 43}$, giving the range of CHF in microgravity as (3.8 8.4) $\times 10^{5} \mathrm{~W} / \mathrm{m}^{2}$. In ground experiments, a new steady nucleate boiling state can be maintained for the whole duration of about 30 seconds when the set-point of the heater temperature changed upward from $T_{\mathrm{W}, 42}$ to $T_{\mathrm{W}, 43}$. Two-mode transition boiling occurs when the set-point of the heater temperature changed upward from $T_{\mathrm{W}, 43}$ to $T_{\mathrm{W}, 44}$. Thus, the CHF, or more accurately, the lower limit of CHF, can be determined as $7.9 \times 10^{5} \mathrm{~W} / \mathrm{m}^{2}$ and $8.4 \times 10^{5} \mathrm{~W} / \mathrm{m}^{2}$ for the ground experiments before and after flight, respectively.

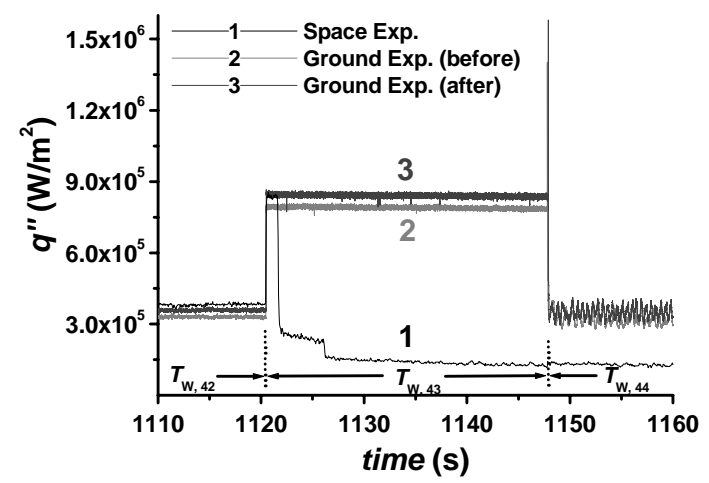

Fig. 3 Variety of heat flux near the CHF in different gravity levels

Fig. 4 plots the results of nucleate boiling obtained in the space and ground experiments. In both the space experiment and ground experiments, the boiling curve of up-stepping process is lower than that of down-stepping process due to the difference of bubble behaviors on the heater surface. It is also shown that except one point of $\Delta T_{\text {sat }}=7.4{ }^{\circ} \mathrm{C}$ during the $2^{\text {nd }}$ up-stepping of temperature, the heat transfer of nucleate pool boiling in microgravity is slightly enhanced comparing with that in normal gravity.

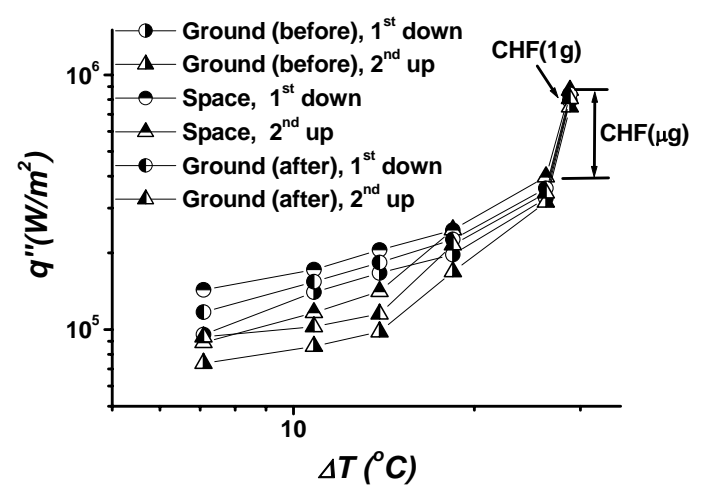

Fig. 4 Result of nucleate boiling

Fig. 5 shows the scaling of CHF with the gravity. It's found that LD-Zuber model, established on the mechanism of hydrodynamic instability, gives a good prediction on the trend of CHF in different gravity conditions, although the value of $R$ ' is far beyond the initial application range of LD-Zuber model. It is contrary to the traditional viewpoint on CHF's scale effect, which is based on the results in normal gravity. A parameter, named as "the Limited Nucleate Size", has been introduced to interpret this phenomenon, which is independent with gravity. A non-dimensional coefficient $R$ is defined as the ratio of the limited nucleate size and the diameter of wire. The occurrence of the CHF will be caused by the mechanism of hydrodynamic instability if $R$ is small, while it will be caused by the mechanism of local dryout if $\mathcal{R}$ is large. Further researches are needed for the delimitation of the two mechanisms.

\section{Bubble dynamics in boiling}

Fig. 6 shows the typical process of coalescence, vibration and departure of bubblers 
in the regime of fully developed nucleate pool boiling. It is found that the vibration due to coalescence of adjacent bubbles is the primary reason of bubble departure in microgravity in this regime. On the contrary, distinct bubble behaviors are observed in isolated bubble regime of nucleate pool boiling in microgravity (Fig. 7), comparing with those in normal gravity.

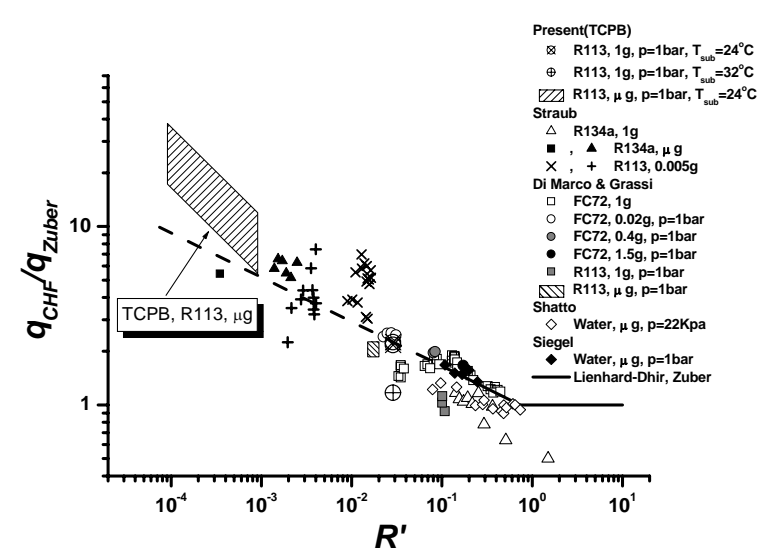

Fig. 5 Scaling of CHF with gravity

Fig. 8 plots the statistical data of isolated bubbles which appeared in the central section of the wire and departing along the direction paralleled to the observing plane. It is found that three critical bubble diameters, namely $D_{b 1}=0.3 \mathrm{~mm}, D_{b 2}=3.5 \mathrm{~mm}, D_{b 3}=8.5 \mathrm{~mm}$, are observed in the space experiments, which divide the bubbles into four groups. The smallest bubbles with diameter less than $0.3 \mathrm{~mm}$ are continually forming and growing on the heater surface before departing slowly from the wire when their sizes exceed some critical value $(0.3$ $\mathrm{mm})$. The bigger bubbles with diameter between $3.5 \mathrm{~mm}$ and $6.6 \mathrm{~mm}$ stay on the wire, oscillate along the wire, and coalesce with adjacent bubbles. The biggest bubble with diameter of the order of $10 \mathrm{~mm}$, which is yielded during the onset of boiling, stays continuously on the wire and swallows continually up adjacent small bubbles till its diameter exceeds $8.5 \mathrm{~mm}$ at the critical heat flux (CHF). The behaviors of small bubbles are observed both in microgravity and in normal gravity, while the last two kinds of bubble behaviors are observed only in microgravity aboard the satellite.

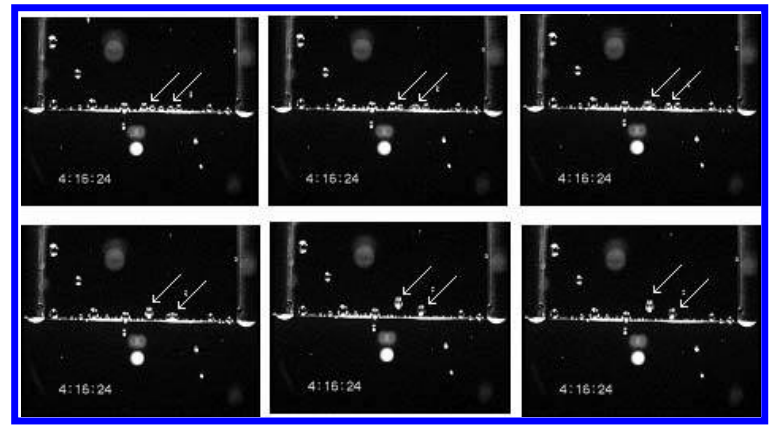

Fig. 6 Coalescence, vibration and departure of bubblers.

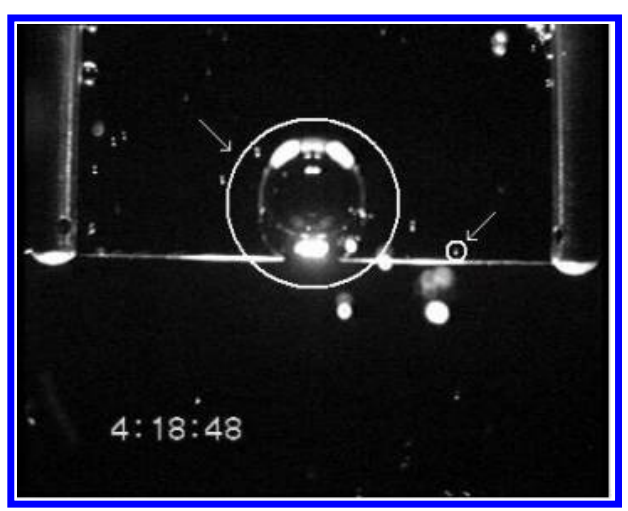

Fig.7 Behaviors of isolated bubbles

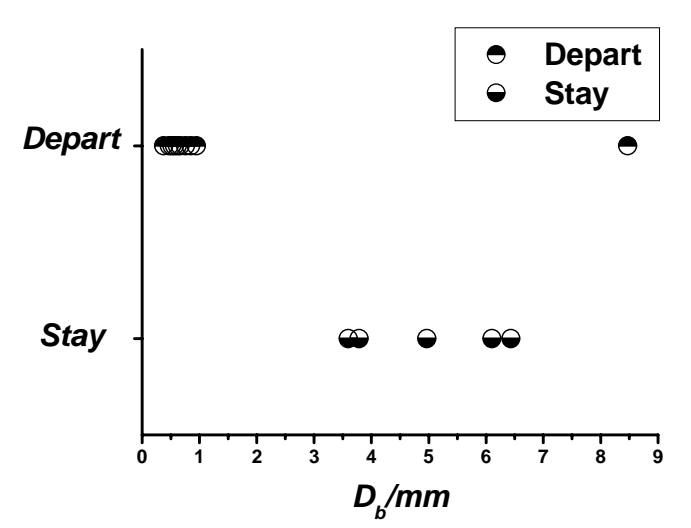

Fig. 8 Bubble departure diameters

Presently, no model can predict the above observation. For example, the models of Karri (1988) and Lee (1992) predict respectively the 
departure diameter as $0.28 \mathrm{~mm}$ and $0.13 \mathrm{~mm}$, close to the $1^{\text {st }}$ bubble departure diameter, while the models of Fritz $(1935,1936)$ and Zeng et al. (1993) predict respectively the departure diameter as $10.9 \mathrm{~mm}$ and $7.1 \mathrm{~mm}$, close to the $2^{\text {nd }}$ bubble departure diameter observed in the present study. The reason may be that the Marangoni effect is ignored in these models. In microgravity environment, the interface effect will become a dominant factor because the buoyancy effect is extremely restrained and even eliminated. Commonly, the increase of temperature will lead the decrease of surface tension of liquid, thus the uneven temperature field will cause tangential forces that act on the interface, which pull the bulk fluid moving. This motion is usually called Marangoni convection. During the process of subcooled pool boiling, the temperature of the heater surface is higher than the saturation temperature, and the temperature of subcooled liquid far away from the heater surface is lower than the saturation temperature. Therefore, a distinct temperature gradient will be formed in the liquid around the bubble. Generally, the surface tension decreases as the increase of temperature. Then the Marangoni force will pull the bubble moving towards the high temperature side (namely the heater surface), which prevents the bubble from departing from the heater's surface. So the Marangoni force should be considered as the resistant force $\left(F_{R}\right)$.

Consider the bubble Marangoni migration in the bulk liquid with a constant temperature gradient. If the wall effect and the variety of physical properties are ignored, the bubble Marangoni migration will finally reach a steady condition when the force due to Marangoni effect is balanced by the viscous drag force acting on the bubble. Therefore, the Marangoni force can be expressed as follows using the linear Stocks theory for the case of infinitesimal
Reynolds and Marangoni numbers (Batchelor 1994, Young et al. 1959) as

$$
F_{m}=2 K \pi\left|\sigma_{T}\right| T^{\prime} R^{2}
$$

where $\sigma_{T}, R$ and $T$ denote the temperature coefficient of surface tension, the bubble radius, and the temperature gradient, respectively. The empirical coefficient $K$ is used to modify the departure from the linear theory for the case of finite Reynolds and Marangoni numbers

Other forces acting on the bubble can be expressed followed by Lee (1992). Except the Marangoni force, the buoyancy force $F_{b}$, the inertia force $F_{i}$, and the pressure force $F_{p}$ should be considered as the departure forces $\left(F_{D}\right)$ since they pull the bubble to depart from the heater surface, while the drag force $F_{d}$ and the surface tension force $F_{S}$ should be considered as the resistant forces $\left(F_{R}\right)$ since they prevent the bubble from departing from the heater's surface. Therefore, the sum of the forces acting on the bubble is,

$$
F=F_{D}-F_{R}=\left(F_{i}+F_{p}+F_{b}\right)-\left(F_{d}+F_{s}+F_{m}\right)
$$

Following Karri (1988) and Lee (1992), an asymptotic bubble growth relation can be assumed

$$
R=E \tau^{1 / 2}
$$

where the empirical parameter $E$ is calculated by the relation given by Cole \& Shulman (1966)

$$
E=\frac{1}{2 \sqrt{\pi}} J a \sqrt{\alpha_{l}}
$$

where, $J a$ and $\alpha_{l}$ are the Jacob number and the heat diffusivity coefficient of liquid.

In this way, formula (2) can be rewritten as

$$
f(y)=C_{4} y^{4}+C_{3} y^{3}+C_{1} y+C_{0}
$$

where,

$$
\begin{aligned}
& \mathrm{y}=\tau^{1 / 2} \\
& C_{4}=\frac{4}{3} \pi E^{3}\left(\rho_{L}-\rho_{V}\right) g
\end{aligned}
$$




$$
\begin{aligned}
& C_{3}=-2 K \pi\left|\sigma_{T}\right| T_{1} E^{2} \\
& C_{1}=4 \sigma R_{0} \sin ^{2} \beta+\frac{\pi}{3} \rho_{L} E^{4} \\
& C_{0}=R_{0} E^{3} \rho_{L} \sin ^{2} \beta\left(\frac{1}{3}-\frac{3}{8} C_{d}\right)
\end{aligned}
$$

According to Eq. (2) and Eq. (5), the following conclusion can be obtained: If $f(y)<0$, the departure force is larger than the resistant force, so the bubble will stay on the heater's surface; if $f(y)>0$, the departure force is smaller than the resistant force, so the bubble will depart from the heater's surface.

Using the following parameters for $\mathrm{R} 113$ are used, $p_{l}=0.1 \mathrm{MPa}, \Delta T_{\text {sub }}=24^{\circ} \mathrm{C}, \Delta T_{\text {sat }}=30^{\circ} \mathrm{C}$, $\beta=5^{\circ}, K=0.035$, the varieties of $f(y)$ with gravity are plotted in Fig. 9, where the abscissa is replaced by the bubble diameter with Eq. (3).

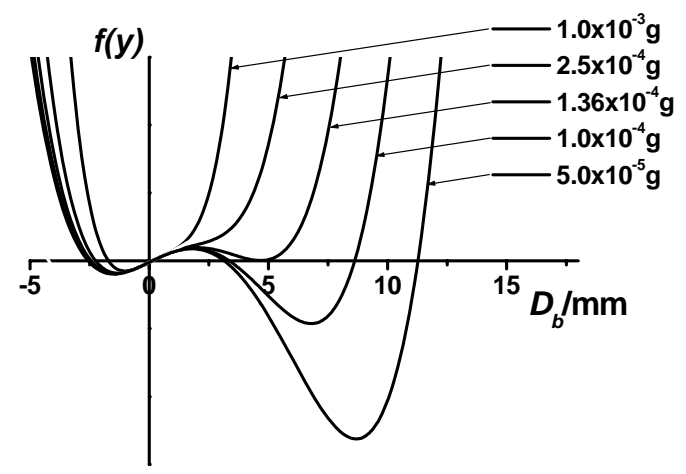

Fig. 9 Variety of $f(y)$ with gravity

Considering the positive half plane of $D_{b}$, there is only one point of intersection between Eq. (5) and the abscissa, i.e. $D_{b 1} \approx 0.11 \mathrm{~mm}$, in normal gravity. It is very close to that predicted by Lee's model, which shows that the effect of Marangoni convection can be ignored in normal gravity. This value is gradually increasing as the residual gravity decreasing, although the increment is very tiny. On the contrary, when the gravity reduces to $1.36 \times 10^{-4} \mathrm{~g}$, a second point $D_{b 2}$ of intersection between Eq. (5) and the abscissa appears. Furthermore, when the gravity is less than $1.36 \times 10^{-4} \mathrm{~g}$, there are three points of intersection $\left(D_{b 1}, D_{b 2}\right.$, and $\left.D_{b 3}\right)$ between Eq. (5) and the positive abscissa, which divide the positive abscissa into four regions:

1) $D_{b}<D_{b 1}, f(y)<0$, the bubble stays on the heater surface;

2) $D_{b 2}>D_{b}>D_{b 1}, f(y)>0$, the bubble departs from the heater surface;

3) $D_{b 3}>D_{b}>D_{b 2}, f(y)<0$, the bubble stays on the heater surface; and

4) $D_{b}>D_{b 3}, f(y)>0$, the bubble departs from the heater surface.

It agrees qualitatively with the observation in space experiment. Furthermore, a quantitative agreement can also be obtained with $K=0.035$, in which $D_{b 1}=0.11 \mathrm{~mm}, D_{b 2}=3.4 \mathrm{~mm}, D_{b 3}=8.6 \mathrm{~mm}$ are predicted.

\section{Conclusion}

A temperature-controlled pool boiling (TCPB) device has been developed to study the bubble behavior and heat transfer in subcooled pool boiling phenomenon both in normal gravity and in microgravity. The space experiment has been performed aboard the $22^{\text {nd }}$ Chinese recoverable satellite, while the ground experiments have also been conducted before and after the flight.

Three modes of heat transfer, namely single-phase natural convection, nucleate boiling, and two-mode transition boiling, are observed both in normal gravity and in microgravity. The analysis of single phase convection verifies the reliability of experimental facility and confirms the residual gravity level on the satellite as $10^{-3} \sim 10^{-5} \mathrm{~g}$. It is found that the temperature of the onset of boiling is independent of the gravity level. It's also found that the heat transfer of nucleate pool boiling is slightly enhanced in microgravity comparing with those in normal gravity. It's also found that the correlation of 
Lienhard and Dhir (1973) can predict the CHF with good agreement, although the range of the dimensionless radius is extended by three or more decades above the originally set limit.

On the contrary, distinct bubble behaviors are observed in isolated bubble regime of nucleate pool boiling in microgravity, comparing with those in normal gravity. Three critical bubble diameters are observed in microgravity, which divide the observed vapor bubbles into four regimes with different sizes. Considering the Marangoni effect, a qualitative model is proposed based on Lee's model to reveal the mechanism underlying the bubble departure processes and a quantitative agreement can also be acquired.

\section{Acknowledgements:}

The present study is supported financially by the Chinese Academy of Sciences under the grant of KJCX2-SW-L05, and the National Natural Science Foundation of China under the grant of 10432060 . The authors also wish to acknowledge the fruitful discussion with Prof. H. Ohta (Kyushu University, Japan).

\section{References:}

[1] Batchelor, KG. 1994. An Introduction to Fluid Dynamics. Cambridge University Press.

[2] Cole R, Shulman HL. 1966. Bubble growth rates at high Jakob numbers. Int. J. Heat Mass Transfer, 9: 1377-1390.

[3] Di Marco P, Grassi W. 2002. Motivation and results of a long-term research on pool boiling heat transfer in low gravity. Int. $J$. Therm. Sci., 41(7): 567-585.

[4] Fritz W, Ende W. 1935. Berechnung des maximalvolumens von dampfslasen. Physik, 36: 379-384.

[5] Fritz W, Ende W. 1936. Uber den verdampfungsvorgang nach kinematographischen aufnahmen an dampfblasen. Physik, 37: 391-401.

[6] Karri SBR. 1988. Dynamics of bubble depature in micro-gravity. Chem. Eng. Comm., 70: 127-135.

[7] Kuehn TH, Goldstein RJ. 1976. Correlating equations for natural convection heat transfer between horizontal circular cylinders. Int. J. Heat Mass Transfer 19: 1127-1134.

[8] Lee DJ. 1992. "Bubble departure radius under microgravity." Chem. Eng. Comm., 117: 175-189.

[9] Straub J. 2001. Boiling heat transfer and bubble dynamics in microgravity. $A d v$. Heat Transfer, 35: 57-172.

[10] Wan SX, Zhao JF, Liu G, Hu WR. 2003. TCPB device: description and preliminary ground experimental results. In: 54th Int. Astronautical Cong., Sep. 29-Oct 3, Bremen, Germany.

[11] Young NO, Goldstein JS, Block MJ. 1959. The motion of bubbles in a vertical temperature gradient. J. Fluid Mech. 6: 350-356.

[12] Zeng LZ, Klausner JF, Mei R. 1993. A unified model for the prediction of bubble detachment diameters in boiling system - 1 . pool boiling. Int. J. Heat Mass Transfer, 36: 2261-2270. 